\title{
Neurobehavioral Performance in Feline Immunodeficiency Virus Infection: Integrated Analysis of Viral Burden, Neuroinflammation, and Neuronal Injury in Cortex
}

\author{
Ferdinand Maingat, ${ }^{1}$ Pornpun Vivithanaporn, ${ }^{1}$ Yu Zhu, ${ }^{1}$ Andrew Taylor, ${ }^{2}$ Glen Baker, ${ }^{3}$ Keir Pearson, ${ }^{2}$ and \\ Christopher Power ${ }^{1,3}$ \\ Departments of ${ }^{1}$ Medicine, ${ }^{2}$ Physiology, and ${ }^{3}$ Psychiatry, University of Alberta, Edmonton, Alberta T6G 2S2, Canada
}

\begin{abstract}
Human immunodeficiency virus (HIV) infection causes motor and neurocognitive abnormalities affecting $>50 \%$ of children and $20 \%$ of adults with HIV/AIDS (acquired immunodeficiency syndrome). The closely related lentivirus, feline immunodeficiency virus (FIV), also causes neurobehavioral deficits. Herein, we investigated the extent to which FIV infection affected specific motor and cognitive tasks in conjunction with viral burden and immune responses within the brain. Neonatal animals were infected with a neurovirulent FIV strain (FIV-Ch) and assessed in terms of systemic immune parameters, viral burden, neurobehavioral performance, and neuropathological features. FIV-infected animals displayed less weight gain and lower blood CD $4{ }^{+}$T-cell levels than mock-infected animals $(p<0.05)$. Gait analyses disclosed greater gait width with increased variation in FIV-infected animals $(p<0.05)$. Maze performance showed that FIV-infected animals were slower and made more navigational errors than mock-infected animals $(p<0.05)$. In the object memory test, the FIV-infected group exhibited fewer successful steps with more trajectory errors compared with the mock-infected group $(p<0.05)$. Performance on the gait, maze, and object memory tests was inversely correlated with $F 4 / 80$ and $C D 3 \varepsilon$ expression $(p<0.05)$ and with viral burden in parietal cortex $(p<0.05)$. Amino acid analysis in cortex showed that $\mathrm{D}$-serine levels were reduced in FIV-infected animals, which was accompanied by diminished kainate and AMPA receptor subunit expression $(p<0.05)$. The neurobehavioral findings in FIV-infected animals were associated with increased gliosis and reduced cortical neuronal counts $(p<0.05)$. The present studies indicated that specific motor and neurocognitive abilities were impaired in FIV infection and that these effects were closely coupled with viral burden, neuroinflammation, and neuronal loss.
\end{abstract}

\section{Introduction}

A signature feature of lentivirus infections, including human (HIV), simian (SIV), and feline (FIV) immunodeficiency viruses, is that they cause neurological disease, likely because of infection of the nervous system coupled with progressive immunosuppression (Patrick et al., 2002). Although the spectrum of neurological disease phenotypes associated with HIV infection is broad and growing, the principal neurobehavioral phenotypes in animal lentivirus infections remain mostly analyzed using qualitative assays. Indeed, among HIV/acquired immunodeficiency syndrome (AIDS) patients, the neurological phenotypes involving the CNS often escape clinical recognition because they are overlooked on routine evaluations or eclipsed by other (overwhelming) systemic clinical problems. Nonetheless, the prototypic CNS syndromes caused by HIV infection, HIV-associated dementia, and

\footnotetext{
Received Dec. 5, 2008; revised April 2, 2009; accepted May 7, 2009.

These studies were supported by the Canadian Institutes of Health Research. K.P. holds a Canada Research Chair (CRC) (Tier 1) in Movement Physiology, G.B. holds a CRC (Tier 1) in Neurochemistry and Drug Development, and C.P. holds a CRC (Tier 1) in Neurological Infection and Immunity and an Alberta Heritage Foundation for Medical Research Senior Scholarship. We thank Leah DeBlock for assistance with manuscript preparation.

Correspondence should be addressed to Dr. Christopher Power, Department of Medicine (Neurology), 6-11 Heritage Medical Research Centre, University of Alberta, Edmonton, AB T6G 252, Canada. E-mail: chris.power@ualberta.ca.

DOI:10.1523/JNEUROSCI.5818-08.2009

Copyright $\odot 2009$ Society for Neuroscience $\quad$ 0270-6474/09/298429-09\$15.00/0
}

the antecedent condition, minor neurocognitive disorder, are defined by motor, behavioral, and cognitive dysfunctions (McArthur et al., 2005). Similarly, aberrant motor function appears to characterize animal lentivirus infections, albeit defined in limited terms (Murray et al., 1992; Power et al., 1998).

FIV is a member of the lentivirus subfamily that causes persistent infection in domestic cats and shares many of the immunological and neurological pathogenic aspects with HIV and SIV in their respective hosts (Bendinelli et al., 1995). Sharing structural and biochemical properties with HIV and SIV, FIV causes neurological abnormalities in $20-40 \%$ of naturally infected cats by entering the CNS and infecting astrocytes, parenchymal microglia, and perivascular macrophages (Power, 2001). Neuropathogenic effects mediated by FIV infection are viral strain specific (Power et al., 1998; Johnston et al., 2002) and vary from seizures, ataxia, psychomotor retardation, aggressivity, disrupted sleep and arousal patterns to stereotypic motor behaviors (Phillips et al., 1994; Prospéro-García et al., 1999; Steigerwald et al., 1999). As with HIV and SIV infections, neuroimmune activation occurs in FIV, including microglial and astrocytic activation, leukocyte infiltration with the ensuing production of cytokines, chemokines, proteases, and other inflammatory factors in the CNS (Power et al., 1997; Poli et al., 1999; Noorbakhsh et al., 2006). These neuroinflammatory changes are accompanied by neuronal injury and loss in the brain, especially in the basal ganglia and cortical 
Table 1. Primers used for real-time RT-PCR analyses

\begin{tabular}{|c|c|c|c|}
\hline Primer name & Sequence $\left(5^{\prime}-3^{\prime}\right)$ & $T_{\mathrm{m}}\left({ }^{\circ} \mathrm{C}\right)$ & Concentration \\
\hline GAPDH forward & AGC CTT CTC CAT GGT GGT GAA & $56-60$ & $5 \mu \mathrm{M}$ \\
\hline GAPDH reverse & CGG AGT CAA CGG ATT TGG & $56-60$ & $5 \mu \mathrm{M}$ \\
\hline CD3 $\in$ forward & AAG CAA GAG TGT GTC AGA ACT & 60 & $5 \mu \mathrm{M}$ \\
\hline$C D 3 \in$ reverse & CTG ATT CAG GCC AGA ATA CAG & 60 & $5 \mu \mathrm{M}$ \\
\hline F4/80 forward & CAC GAC GGA GTT ACC CTT GT & 56 & $5 \mu \mathrm{M}$ \\
\hline $\mathrm{F} 4 / 80$ reverse & GCG AGG AAA AGG TAG TGC AG & 56 & $5 \mu \mathrm{M}$ \\
\hline GFAP forward & CTC ACC ATC CCG CAT CTC CAC AGT & 56 & $5 \mu \mathrm{M}$ \\
\hline GFAP reverse & GGA CAT CGA GAT CGC CAC CTA CAG & 56 & $5 \mu \mathrm{M}$ \\
\hline GluR1 forward & GGC ATC GGC TAC CAC TAC AT & 60 & $5 \mu \mathrm{M}$ \\
\hline GluR1 reverse & GTT CTT CCA CTG CTG CAT GA & 60 & $5 \mu \mathrm{M}$ \\
\hline GluR5 forward & CTG GCA GTG GCT CCT CTA AC & 60 & $5 \mu \mathrm{M}$ \\
\hline GluR5 reverse & GCT TCC GGT AGA GAA TGC TG & 60 & $5 \mu \mathrm{M}$ \\
\hline KA2 forward & TGG GGA TCA GCA TCC TCT AC & 60 & $5 \mu \mathrm{M}$ \\
\hline $\mathrm{KA} 2$ reverse & GCG AGG TAG GCG AGA AGC ATG AA & 60 & $5 \mu \mathrm{M}$ \\
\hline
\end{tabular}

regions, which are the major sites of infection and immune activation (Zink et al., 2006; Fletcher et al., 2008).

Although previous studies have highlighted individual aspects of the neuropathology of FIV infection (Ryan et al., 2005; Meeker, 2007) and conducted qualitative neurobehavioral studies (Steigerwald et al., 1999), no study has integrated both these facets of lentiviral infection in a quantitative manner and related it to host immune response or viral load. Given the above observations, we tested the hypothesis that multiple neurobehavioral tasks are impaired in FIV infection and associated with different components of the neuroimmune response(s) to lentivirus infection of the brain. Our studies showed that FIV infection resulted in both cognitive and motor deficits, which were correlated with selective activation of certain cell types in the brain, viral copy number, and neuronal loss.

\section{Materials and Methods}

Animals and virus inoculation

Adult specific pathogen-free pregnant cats (queens) were housed according to Universities of Alberta and Calgary animal care facilities' guidelines and in agreement with Canadian Council on Animal Care (CCAC) guidelines. All queens were negative for feline retroviruses (FIV; feline leukemia virus) by PCR analysis and serological testing. At day 1 postnatal, animals (FIV+) were inoculated (right frontal lobe) with $200 \mu \mathrm{l}$ of FIV-Ch2 29 at $10^{4} 50 \%$ tissue culture infective dose/ $\mathrm{ml}(n=15)$ using a 30 gauge needle and syringe via intracranial injection in accordance with CCAC guidelines, as described previously (Power et al., 1998; Johnston and Power, 2002). Mock-infected (FIV-) animals received heatinactivated virus $(n=13)$. Cats were weaned at 6 weeks and monitored for 12 or 15 weeks, during which body weight and behavioral performance were assessed weekly and blood samples were taken for analyses of CD4/CD8 ${ }^{+}$T-cell populations. At 12 and 15 weeks, the animals were killed by pentobarbital overdose; brain and plasma were harvested at this time. Samples were frozen immediately by immersion in liquid nitrogen for subsequent protein or total RNA extractions. Brain tissue from the left hemisphere was fixed in $4 \%$ buffered, $\mathrm{pH} 7.4$, paraformaldehyde for immunofluorescence studies.

\section{Behavioral tests}

Gait analysis. Gait was assessed by inking the rear footpads and luring the animals across a suspended 6-inch-wide by 8 -foot-long plank covered with a sheet of paper. Only trials with a continuous forward walking motion across the plank were scored but repeated in triplicate. A straight line bisecting the left and right footprints was drawn down the length of the paper (see Fig. $2 \mathrm{~A}$ ). The distance between two lines (running parallel to the bisecting line) originating from the middle of each footprint (right and left) was measured as the gait width. The gait width of a cat consisted of an average of a minimum of 10 of these measurements. The distance between the shoulder blades of a cat in a scruff restraint was also mea- sured and termed "chest width." The measurement of gait was assessed as the cat's average gait width compared with its chest width as well as the $\mathrm{SD}$ or variance in gait width.

Maze test. A modified T maze (Levine et al., 1987) was used to test spatial memory, cognitive learning capacity, and performance speed. The maze was constructed from Plexiglas measuring 4 feet wide by 5 feet long by 8 inches high (see Fig. 3A, left). The walls were opaque and the lid was transparent to observe the movement of the cats. The gray lines represent moveable doors to close and open sections of the maze. For the first three sessions, animals were adapted to the maze with all of the doors open to permit exploration and facilitate habituation. Three training sessions were conducted on separate days in which either the whole left side (see Fig. $3 A$, middle) and right sides (see Fig. $3 A$, right) of the maze and any dead ends were closed off, thus requiring the cats to learn the fastest way through the maze. For each training session, 10 consecutive trials were conducted for each side. Animals were fasted overnight before training days and were trained to walk through the maze to obtain food. On testing days, a minimum of eight trials was collected for each animal, during which the total time to enter and exit the maze and errors in navigation were determined. A timer was started once the cat started forward movement after being placed in the maze opening and was stopped immediately on the animal exiting. During testing, all doors in the maze were open (see Fig. $3 A$, left) and errors in navigation were scored as instances in which the cat had turned into a dead end, if the animal reversed motion (when not required), and if the animal continued past the exit and turned back into the maze. After each successful trial, animals were fed.

Object memory test. Cats were fitted with reflective markers on their left and right hindpaws and trained to walk down a narrow alley toward a 6-cm-high removable barrier to obtain food (see Fig. 4Ai-iv) (McVea and Pearson, 2006). The food was positioned such that the cat would have to step over the barrier with only their forelimbs to obtain the food (see Fig. $4 A i, i i$ ). While the cat was distracted by the food, the barrier was lowered (see Fig. 4Aiii), and after $10 \mathrm{~s}$ the food was moved forward, luring the cat forward (see Fig. 4 Aiv). Trials were recorded on video and analyzed with Peak Motus software using the reflective markers on the hindpaws and the top of the barrier as reference points. Maximum step height and trajectory were recorded for a series of 10 trials in which the barrier was dropped down and four control trials in which the barrier was unmoved and the cat was allowed to step over the barrier continuously without stopping for food.

\section{Plasma and neural tissue viral load}

A real-time reverse transcriptase (RT)-PCR protocol was developed in which the oligonucleotide primers were derived from the FIV pol gene and were used to determine the number of copies of viral RNA in plasma and brain tissue (per microgram of RNA) (Zhu et al., 2007). To obtain a standard curve, in vitro RNA transcripts of the FIV pol region were generated from pBS-FIV pol. This plasmid contained a $3.1 \mathrm{~kb}$ pol gene fragment (positions 2137-5282) generated from the FIV molecular clone, p34TF10, by PCR using primers (POL-KpnI, GAA AAT GGT ACC CAA AAT ATG ATT GG; POL-XhoI, CTC CTC CTC GAG CAC TGC AAA G) that introduced unique $\mathrm{Kp} n \mathrm{I}$ and $\mathrm{XhoI}$ restriction sites at the $5^{\prime}$ and $3^{\prime}$ ends of the amplicon, respectively. The PCR fragment was digested with $K p n \mathrm{I}$ and $\mathrm{XhoI}$ and the fragment was ligated into the $K p n \mathrm{I}$ and $\mathrm{XhoI}$ sites downstream of the T7 promoter in pBluescript SK II(+) resulting in pBS-FIV pol. Correct insertion of the fragment was confirmed by restriction enzyme analysis and sequencing. The plasmid was linearized with XhoI, treated with proteinase $\mathrm{K}\left(0.2 \mathrm{mg} / \mathrm{ml} ; 0.5 \% \mathrm{SDS} ; 50^{\circ} \mathrm{C}\right.$ for $\left.30 \mathrm{~min}\right)$, and purified by phenol:chloroform extraction and ethanol precipitation. An aliquot of $2-3 \mu \mathrm{g}$ of linearized DNA was used as a template for in vitro transcription to generate pol transcripts using a T7 in vitro RNA transcription kit (Ambion). RNA transcripts were purified by phenol:chloroform extraction and isopropanol precipitation. The amount of RNA was measured by spectrophotometry and the concentration was converted to copies per microgram of RNA. A standard curve was generated with serial 10 -fold dilutions $\left(10^{11}\right.$ copies/8 $\mu \mathrm{l}$ to 1 copy/8 $\mu \mathrm{l}$ ) of the in vitro-transcribed RNA. Subsequently, the transcripts were treated with RNase-free DNaseI (Promega) and reverse-transcribed using Super- 

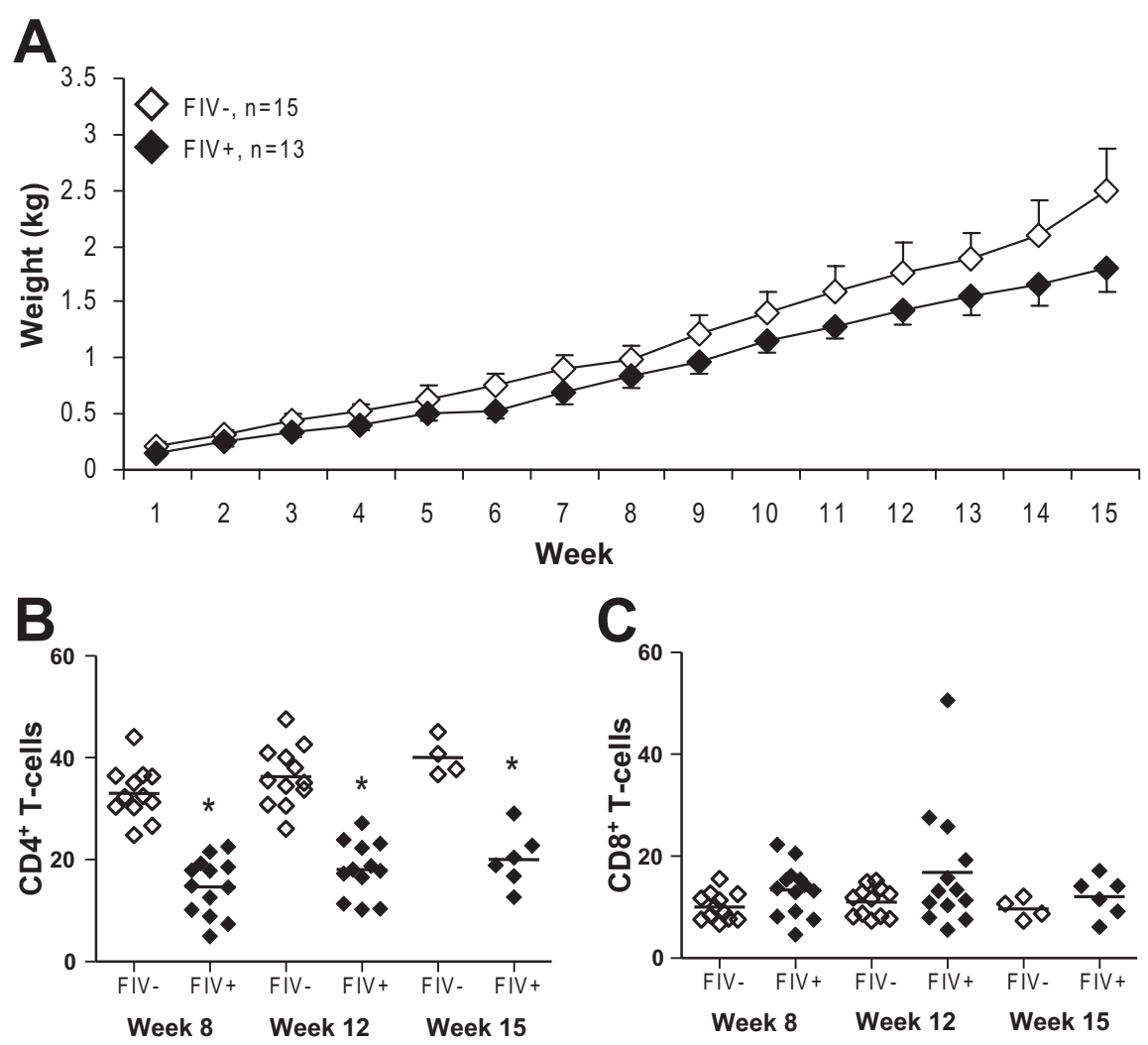

Figure 1. FIV infection causes reduced weight gain and immunosuppression. Weight gain was significantly retarded in FIV+ animals compared with FIV - animals at all time points after infection except week $1(\boldsymbol{A})$. FIV infection reduced CD4 ${ }^{+}$T-cell levels in blood of infected animals at 8,12 , and 15 week time points $(\boldsymbol{B})$ compared with uninfected animals with minimal change in $\mathrm{CD}^{+}{ }^{+}$T-cell levels (C). Shown are mean \pm SD. ${ }^{*} p<0.05$.

scriptII and random $\mathrm{N}_{6}$ oligomers to prime cDNA synthesis (Invitrogen). RNA was extracted by spin column (RNeasy Minikit; QIAGEN) from plasma and brain, and subsequently cDNA synthesis was performed. The cDNA was diluted 1:2 with sterile water and $5 \mu \mathrm{l}$ were used per PCR. Real-time quantitative PCR was performed using a single-color real-time PCR detection system (Bio-Rad) with each $25 \mu$ l reaction containing $5 \mu \mathrm{l}$ of cDNA (standard or sample), $13.5 \mu \mathrm{l}$ of iQ SYBR Green Supermix (Bio-Rad), $6.5 \mu \mathrm{l}$ of $\mathrm{H}_{2} \mathrm{O}$, and $1 \mu \mathrm{l}$ of primer mix ( $5 \mu \mathrm{M}$ each primer). The FIV-pol oligonucleotide primers (forward primer, $5^{\prime}$-ACC TAC TTC TAG AGA AGC CTG G-3'; reverse primer, 5' -GTA TCT GTC CAA TAG GCT GC- $3^{\prime}$ ) were used to yield a 214 bp product. The amplification protocol consisted of an initial denaturation step of $3 \mathrm{~min}$ at $95^{\circ} \mathrm{C}$, followed by 45 cycles of $95^{\circ} \mathrm{C}$ for $30 \mathrm{~s}, 58^{\circ} \mathrm{C}$ for $30 \mathrm{~s}$, and $72^{\circ} \mathrm{C}$ for $30 \mathrm{~s}$. After a final elongation step of $72^{\circ} \mathrm{C}$ for $5 \mathrm{~min}$ to ensure proper amplification, the amplicons were subjected to a melt curve analysis, in which the temperature was raised from 65 to $99^{\circ} \mathrm{C}$ in $1{ }^{\circ} \mathrm{C}$ increments, and data were acquired for $8 \mathrm{~s}$ at each temperature increment. Viral loads in tissue were expressed as viral RNA copies per microgram of RNA.

\section{Host genes real-time RT-PCR}

First-strand cDNA was synthesized by using aliquots of $1 \mu \mathrm{g}$ of total RNA from cortex and basal ganglia, reverse transcriptase, and random primers (Zhu et al., 2005). Specific genes were quantified by real-time PCR using the i-Cycler IQ system (Bio-Rad). cDNA prepared from total RNA of cultured DRG cells and DRG tissues was diluted 1:1 with sterile water and $5 \mu$ l were used per PCR. The primers used in the real-time PCR are provided (Table 1). Semiquantitative analysis was performed by monitoring in real time the increase of fluorescence of the SYBR Green dye on a Bio-Rad detection system, as previously reported (Power et al., 2003) and expressed as relative fold change (RFC) compared with mockinfected samples.
Flow cytometry analysis

Peripheral blood mononuclear cells (PBMCs) were isolated from blood of FIV - and FIV+ animals at weeks 8,12 , and 15 as previously reported (Zhu et al., 2005). PBMCs were labeled with anti-feline CD4 or anti-feline CD8 monoclonal antibodies, and FITC-conjugated goat anti-mouse IgG1 antibody was used as a secondary. Omitting the primary antibody served as controls. FACS analysis was performed using the FACSCanto (BD Biosciences) flow cytometer. Cells $\left(5 \times 10^{4}\right)$ were analyzed for each sample (Power et al., 1998).

\section{Immunofluorescence}

Immunofluorescent labeling was performed using $5 \mu \mathrm{m}$ paraffin-embedded serial cat brain sections prepared as previously described (Tsutsui et al., 2004). Briefly, coronal brain sections from the left hemisphere at $10 \mathrm{~mm}$ anterior to bregma were deparaffinized and hydrated using decreasing concentrations of ethanol. Antigen retrieval was performed by boiling the slides in $0.01 \mathrm{~m}$ trisodium citrate buffer, $\mathrm{pH}$ 6.0, for $10 \mathrm{~min}$. Sections were blocked in PBS containing $10 \%$ normal goat serum, $2 \%$ bovine serum albumin, and $0.1 \%$ Triton X-100 overnight at $4^{\circ} \mathrm{C}$. The sections were incubated overnight at $4^{\circ} \mathrm{C}$ with antibodies against ionized calcium binding adaptor molecule (Iba-1) (1:200; Wako), glial fibrillary acidic protein (GFAP) (1:200; Dako), and neuronal nuclei antigen (NeuN) (1:200; Millipore Bioscience Research Reagents), washed in PBS, and then incubated with either Cy3-, Alexa 488 -, or Alexa 680-conjugated goat anti-rabbit or mouse (1:2500 dilution; Invitrogen) for $1 \mathrm{~h}$ at room temperature in the dark followed by repeated washing in PBS. The sections were finally mounted with Gelvatol and examined with a Zeiss Axioskop 2 upright microscope and a LSM510 META (Carl Zeiss MicroImaging) confocal laser-scanning microscope and analyzed using LSM 5 Image Browser. The specificity of staining was confirmed by omitting the primary antibody.

\section{HPLC}

The HPLC procedure was adapted from a previously described protocol (Grant et al., 2006). Brain samples were homogenized in 5 vol of ice-cold double-distilled water. An aliquot was mixed with $100 \%$ methanol to give a final dilution of $60 \times$ and then centrifuged at $12,000 \times g$ for $4 \mathrm{~min}$ at $4^{\circ} \mathrm{C}$. A $5 \mu \mathrm{l}$ aliquot of the supernatant was mixed with $5 \mu \mathrm{l}$ of the derivatizing agent $(2 \mathrm{mg}$ of $N$-isobutyryl-L-cysteine and $1 \mathrm{mg}$ $o$-phthaldialdehyde dissolved in $0.1 \mathrm{ml}$ of methanol, followed by addition of $0.9 \mathrm{ml}$ of $0.1 \mathrm{M}$ sodium borate buffer), and then was placed into a sample management system (Waters Alliance 2690XE; Waters Corporation). HPLC separation was achieved on a Symmetry C18 column $(4.6 \times 150 \mathrm{~mm} ; 3.5 \mu \mathrm{m}$ particle diameter $)$ coupled with a guard column of the same stationary phase (Waters Corporation). The column heater was set to $30^{\circ} \mathrm{C}$ and the sample cooler was held at $4^{\circ} \mathrm{C}$. To separate the derivatized amino acids of interest, a gradient was established from equal parts of solvents $\mathrm{A}(850 \mathrm{ml}$ of $0.04 \mathrm{M}$ sodium phosphate buffer and $150 \mathrm{ml}$ of methanol, $\mathrm{pH} 6.2)$ and $\mathrm{B}(670 \mathrm{ml}$ of $0.04 \mathrm{M}$ sodium phosphate buffer, $555 \mathrm{ml}$ of methanol, and $30 \mathrm{ml}$ of tetrahydrofuran, $\mathrm{pH} 6.2$ ) to only solvent $\mathrm{B}$ by $\sim 45 \mathrm{~min}$, with a flow rate of 0.5 $\mathrm{ml} / \mathrm{min}$. The run time was $60 \mathrm{~min}$ for column washout and equilibrium, and $30 \mathrm{~min}$ to elute all compounds. A Waters 2475 fluorescence detector (Waters Corporation) was used to quantify the eluted compounds (excitation, $344 \mathrm{~nm}$; emission, $433 \mathrm{~nm}$ ). 


\section{Neuronal counts}

To assess neuronal viability in cortex of FIV-and mock-infected animals, we counted NeuN-immunopositive neurons in the left parietal cortex and hippocampus at $+10 \mathrm{~mm}$ from bregma. For the parietal cortex, immunopositive cells were counted at $400 \times$ magnification in all layers of the cortex in five separate nonoverlapping fields for each animal. Similarly, the number of neurons was counted in five different fields within the dentate gyrus at $400 \times$ magnification for each animal. The total number of cells was summed for each animal and averaged across groups.

\section{Statistical analysis}

Statistical analyses were performed by Student's $t$ test when comparing two different groups, FIV-infected (FIV+) versus mock-infected (FIV-), using GraphPad Instat, version 3.0 (GraphPad Software). In addition, for each neurobehavioral task, the number of animals in each group whose performance exceeded 1 SD or more of the mean FIV(control) performance was calculated. Linear regression of relative gene expression compared with behavioral test performance was analyzed by Spearman's $r$, a nonparametric measure of correlation. Values of $p<$ 0.05 were considered significant. Linear regression of viral copy number compared with behavioral test performance and host gene expression was analyzed by Pearson regression. Values of $p<0.05$ were considered significant.

\section{Results}

In the present model, weight gain was significantly lower in FIVinfected (FIV + ) than mock-infected (FIV - ) animals, which became more apparent during the course of the studies (Fig. $1 \mathrm{~A}$ ). As in HIV and SIV infections, CD $4{ }^{+}$T-cell levels were significantly depressed in FIV + animals compared with FIV - animals, which was evident by 8 weeks after infection and persisted until the end of the study (Fig. $1 \mathrm{~B}$ ). Conversely, CD8 ${ }^{+}$T-cells were comparatively unaffected by FIV infection, although several FIV + animals at 12 weeks displayed a nonsignificant activation of CD ${ }^{+}$ T-cells. These studies indicated that systemic and immune abnormalities were present in the FIV-infected animals, which persisted throughout the course of the present studies, recapitulating observations of humans infected with HIV.

Among patients with HIV infection, gait is often impaired, leading to falls and reduced ambulation (Power et al., 2002). It has previously been reported that FIV-infected cats exhibited deficits in walking across progressively narrower planks compared with control animals (Steigerwald et al., 1999). Herein, we investigated gait by measuring gait width as well as variation of gait (Fig. 2A) while walking across a single plank of constant width. These studies disclosed that mean gait width was greater in the FIV + than FIV - animals, which achieved statistical significance at 15 weeks (Fig. 2 B). The diversity in mean gait width analyzed as variance in multiple trials within individual animals was greater in the FIV + than in FIV - groups (Fig. 2C). Of interest, both mean gait width and its variance were greater in the 12-week-old FIV - animals than at 15 weeks. This trend, although not achieving statistical significance, might reflect developmental advancement. Thus, gait was quantitatively impaired in FIV + animals, in keeping with the ataxia often observed in HIV/AIDS patients.

Psychomotor and cognitive processing task deficits also represent striking abnormalities among HIV/AIDS patients (Ances and Ellis, 2007). To assess these functions, we developed a maze task permitting measurement of both speed and memory in the present animals (Fig. $3 A$ ). The mean time taken to complete the maze was greater in the FIV + group compared with FIV - at 12 weeks, but not 15 weeks, after infection (Fig. 3B). Likewise, the mean number of errors was significantly less in the FIV - group at both time points relative to the FIV + animals (Fig. $3 C$ ). Thus,

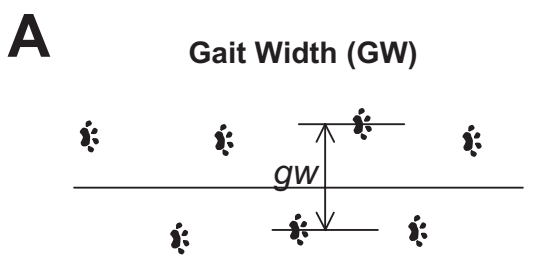

B

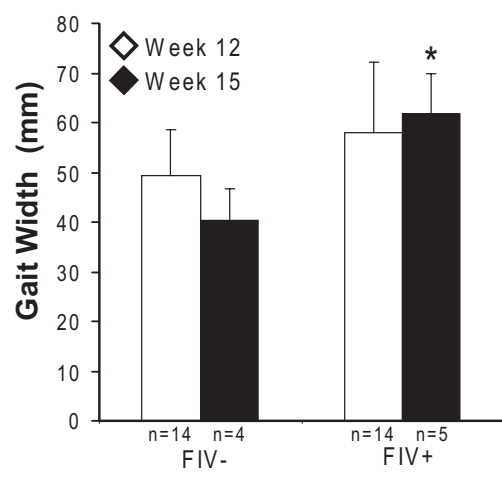

C

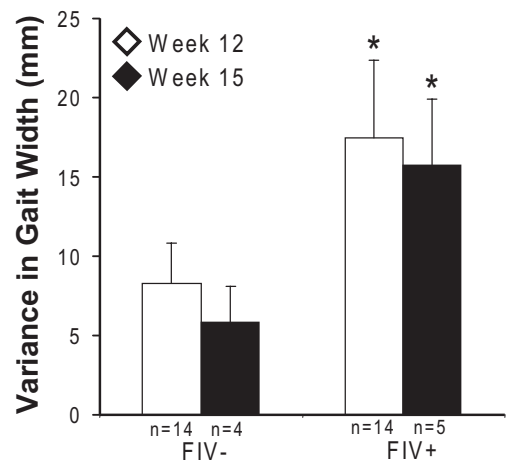

Figure 2. FIV infection diminishes motor performance. FIV + animals exhibited diminished abilities in locomotor tasks at weeks 12 and 15 after infection. Analysis of ink footprints $(\boldsymbol{A})$ made by the cats as they walked across a suspended plank revealed an increase in the gait width of FIV + cats $(\boldsymbol{B})$. Gait was further analyzed by comparing the variance in gait width of FIV + with FIV - cats (C). Shown are mean \pm SD. ${ }^{*} p<0.05$.

the performance rapidity and recall of the maze task was worse in the FIV+ animals.

Memory deficits are among the greatest impediments to patients with HIV infection (McArthur et al., 2005). To evaluate this ability, an object memory task previously developed by McVea and Pearson (2006) was used (Fig. $4 A i-i v$ ). FIV - animals showed an increased mean number of successful trials at both 12 and 15 weeks compared with the FIV+ groups (Fig. 4B). Complementing these observations, the mean percentage number of steps taken by the FIV - animals over the "recalled" bar was greater than in the FIV + animals at 12 weeks after infection but not 15 weeks (Fig. 4C). These findings suggested very specific (cortical) tasks were impaired in the FIV+ groups.

Although there were obvious mean differences between FIV+ and FIV - animals in all tasks, the proportion of animals exhibiting deficits relative to FIV - controls was unclear. Hence the number of animals displaying deficits $>1$ SD beyond the mean FIV - control finding for each task was assessed (Table 2). Depending on the individual task and age, $57-100 \%$ of FIV + animals performed abnormally $(>1 \mathrm{SD})$ on all tasks, whereas 
A

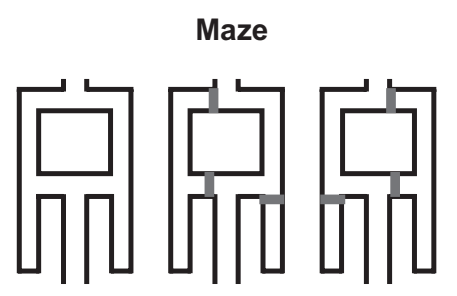

B

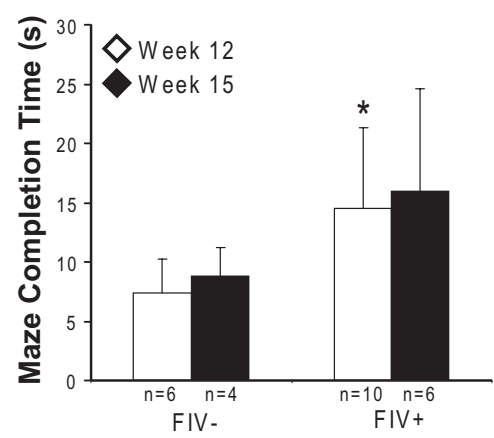

C

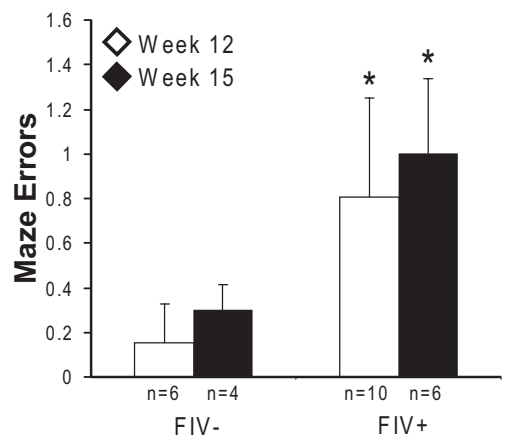

Figure 3. Maze testing demonstrates deficiencies in spatial memory and cognitive abilities. Cats were trained to complete a modified T-maze $(\boldsymbol{A})$ shown with doors closed and were tested for elapsed maze completion times and navigational errors made with the doors open. Maze completion times $(\boldsymbol{B})$ and navigational errors made $(\boldsymbol{C})$ during trials both increased in FIV + cats. Shown are mean \pm SD. ${ }^{*} p<0.05$.

$0-33 \%$ of all FIV - animals showed aberrant performance on corresponding tasks (Table 2). Of note, the effect of sex was also analyzed with respect to each of the neurobehavioral tasks, although there were no significant differences observed among FIV - or FIV + animals based on stratification by sex (data not shown). Nonetheless, these findings underscored the differences between the experimental groups.

To delineate the relationship between these latter tasks and the response of potential immune effector cells, transcript abundance for several genes in brain was assessed at 15 weeks after infection. Mean RFC in CD3e mRNA was greater in the FIV+ than FIV - animals, suggesting that greater T-cell infiltration was present in the FIV-infected animals. Similarly, the monocytoid activation marker, F4/80, exhibited greater expression in the $\mathrm{FIV}+$ animals relative to FIV - animals. However, the inducible astrocyte marker, GFAP, did not display differences in relative expression between FIV - and FIV + groups. The relationship between gene expression and performance on neurobehavioral tests was examined and disclosed that the mean number of successful steps over the recalled barrier was significantly correlated
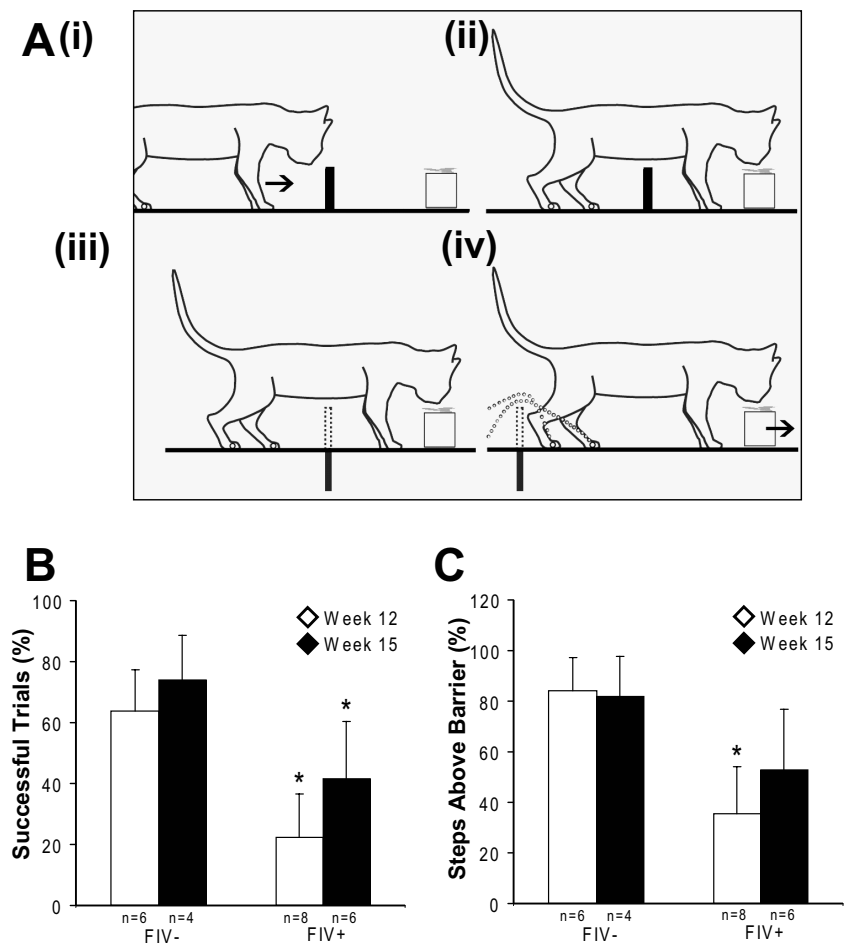

Figure 4. FIV-infected cats show abnormal spatial memory in the object memory test. Cats were lured by food to approach $(\boldsymbol{A} \boldsymbol{i})$ a 6 - $\mathrm{cm}$-high barrier and step over it with their forelimbs (Aii). The gate was lowered and the cat was allowed to eat for $10 \mathrm{~s}$ (Aiii). The food was moved forward to lure the cat to step forward. Reflective dots placed on the outside of the cat's hindlimbs tracked the trajectory and height of the cat's footsteps (Aiv). FIV + cats showed a deficit in recalling the height and position of the barrier as demonstrated by the lower percentage of successful trials, which required a minimum step height and correct trajectory $(\boldsymbol{B})$. The memory deficit in FIV + cats remained evident with the analysis of step height alone (C). Shown are mean \pm SD. ${ }^{*} p<0.05$.

Table 2. Neurobehavioral performance in both experimental groups showing the number (percentage) of animals exhibiting > 1 SD from the mean FIV - (control) performance for each task

\begin{tabular}{llllll}
\hline & Week 12 & & & Week 15 & \\
\cline { 2 - 3 } & FIV - & FIV + & & FIV - & FIV + \\
\hline Gait width & $2 / 14(14.30)$ & $8 / 14(57.10)$ & & $1 / 4(25)$ & $4 / 4(100)$ \\
Gait width variance & $4 / 14(28.60)$ & $13 / 14(92.90)$ & & $1 / 4(25)$ & $4 / 4(100)$ \\
Maze time & $1 / 6(16.70)$ & $8 / 10(80)$ & & $1 / 4(25)$ & $4 / 6(66.70)$ \\
Maze errors & $1 / 6(16.70)$ & $8 / 10(80)$ & & $0 / 4(0)$ & $6 / 6(100)$ \\
OMT successful trials & $1 / 6(16.70)$ & $8 / 8(100)$ & & $0 / 4(0)$ & $4 / 6(66.70)$ \\
OMT steps over obstacle & $2 / 6(33.30)$ & $8 / 8(100)$ & & $0 / 4(0)$ & $4 / 6(66.70)$ \\
\hline
\end{tabular}

with $C D 3 \varepsilon$ (Fig. 5A) and F4/80 (Fig. 5B) but not GFAP (Fig. 5C). Indeed, the performance of several neurobehavioral tasks (Fig. $5 D$ ) was significantly associated with $C D 3 \varepsilon$ and $F 4 / 80$, although not with GFAP expression. Thus individual cellular markers' expression was predictive of neurobehavioral outcomes in this model.

To further define the effects of FIV infection on neurobehavior and host immune response, we investigated the association between viral RNA copy number in cortex or plasma with host immune response and neurobehavioral performance. Analyses of viral burden disclosed that FIV pol sequences were detected in plasma and parietal cortex of FIV + animals only, albeit at lower levels in brain than in plasma (Fig. 6A). Cortical viral burden was significantly correlated with the expression of $C D 3 \varepsilon$ (Fig. $6 B$ ) but not F4/80 (Fig. 6C) or GFAP (Fig. 6D). In addition, viral copy 
numbers in the cortex were associated significantly with maze and object memory tasks but not gait width (Fig. $6 E$ ), implying that viral burden might also be predictive of some neurobehavioral deficits.

Glutamate-mediated neuronal injury is assumed to contribute to lentivirus neuropathogenesis (Kaul and Lipton, 2006), presumably through excitotoxic mechanisms. The measurement of amino acid levels in the cortex revealed that there was a trend toward lower glutamate levels in FIV-infected cats, whereas there was no difference in the levels of other amino acids (e.g., glutamine, taurine, GABA, aspartate, L-serine, and alanine) between FIV+ and FIV- animals (Fig. $7 A, B$ ). Mean levels of $\mathrm{D}$-serine, a potent coagonist at the glycine binding site on the NMDA receptor, were significantly suppressed (Fig. 7B) and the mean glutamate/glutamine ratio was reduced in the FIV+ group (Fig. 7C) $(p<0.05)$. Analyses of ionotropic glutamate receptor transcript levels disclosed that GluR1, GluR5, and KA2 expression was suppressed in the FIV + animals compared with the FIV - group (Fig. 7D). These data suggested that, despite minimal changes in amino acid levels evident in FIV infection, there was disruption of the glutamate-glutamine balance, which may have altered glutamate receptor subunit expression, perhaps reflecting neuronal injury or loss.

Neuropathological studies of the animals showed the presence of Iba-1immunopositive monocytic cells in FIVanimals with few activated cells (Fig. $8 \mathrm{~A}$ ). In contrast, the FIV + animals' brain showed more monocytoid (Iba-Iimmunopositive) cells that were hypertrophied (Fig. $8 \mathrm{~B}$ ). GFAP immunoreactivity did not differ substantially between the FIV - (Fig. $8 B$ ) and FIV+ (Fig. $8 E$ ) experimental groups. However, NeuNimmunopositive neurons were preserved in FIV - animals (Fig. 8C), whereas there were fewer neurons in the FIV + animals (Fig. 8 F). This latter observation was confirmed by a significantly reduced number of neurons in the FIV + animals in the parietal cortex, with a similar trend for hippocampal neurons (Fig. 8G). In summary, the present studies support the notion that neurobehavioral performance in FIV infection is closely coupled with host neuroimmune activation and viral copy number within the brain.

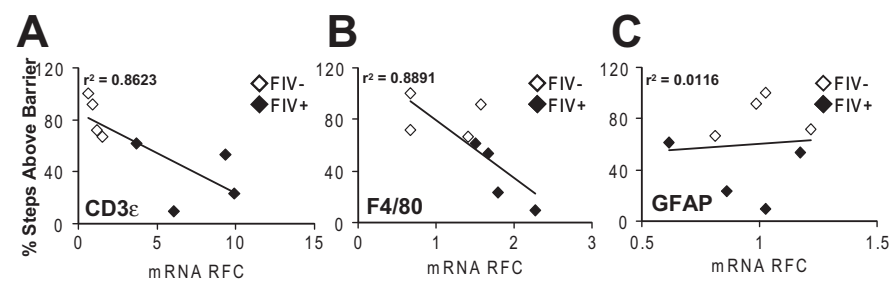

\begin{tabular}{|c|c|c|c|c|c|c|}
\hline & GW & GWV & Maze Time & Maze Errors & OMT Trials & OM T Steps \\
\hline ભొ & 0.3268 & 0.3686 & $0.7346^{*}$ & $0.7201^{*}$ & $0.7762^{* *}$ & $0.8623^{* *}$ \\
\hline $\begin{array}{l}\stackrel{0}{ } \\
\text { 寸 } \\
\text { L }\end{array}$ & $0.6173^{*}$ & 0.4133 & 0.0816 & 0.2235 & $0.8623^{* *}$ & $0.8891^{*}$ \\
\hline 这 & 0.0117 & 0.2545 & 0.0281 & 0.0313 & 0.0023 & 0.0116 \\
\hline
\end{tabular}

Figure 5. FIV infection is associated with neuroimmune activation. Regression analyses of $C D 3 \boldsymbol{\varepsilon}(\boldsymbol{A}), F 4 / 80(\boldsymbol{B})$, and GFAP $(\boldsymbol{C})$ transcript levels compared with percentage of steps above the barrier in the OMT. Spearman regression analysis of neurobehavioral task performance (gait width, gait width variance, maze completion time, maze navigation errors, successful object memory trials, and object memory step heights) with transcript levels of $C D 3 \varepsilon, F 4 / 80$, and GFAP revealed significant $r^{2}$ values in transcript levels of $C D 3 \varepsilon$ and $F 4 / 80$ (D). ${ }^{*} p<0.05 ;{ }^{* *} p<0.01$.
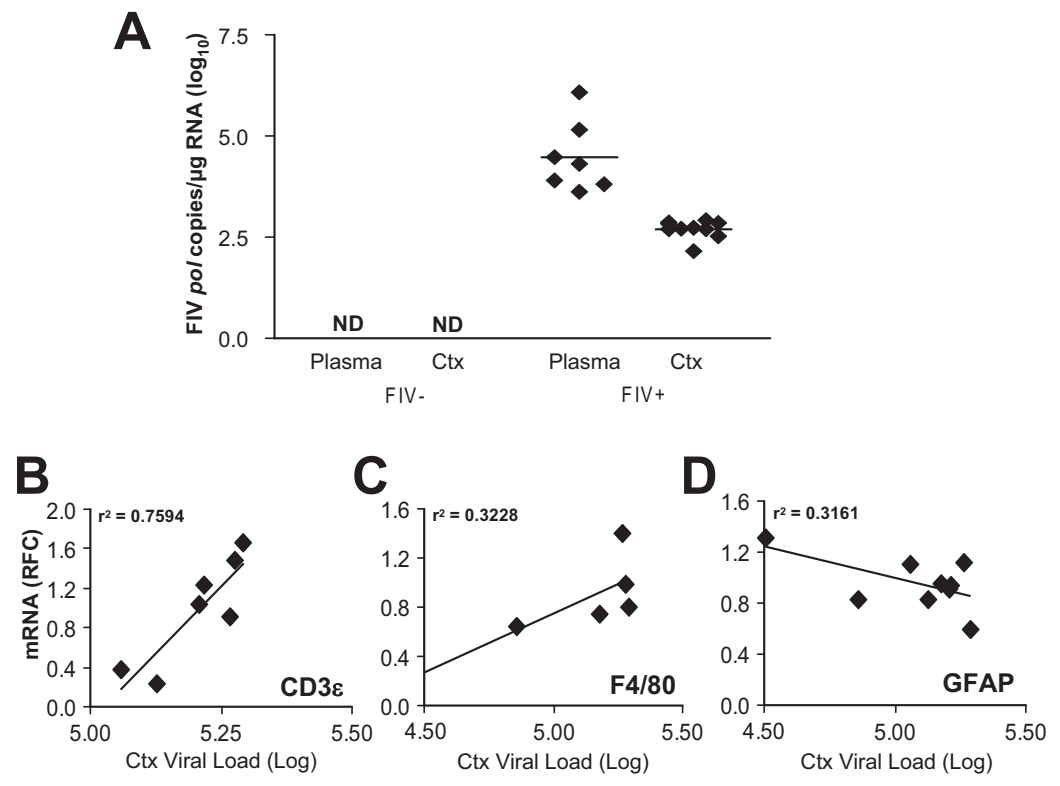

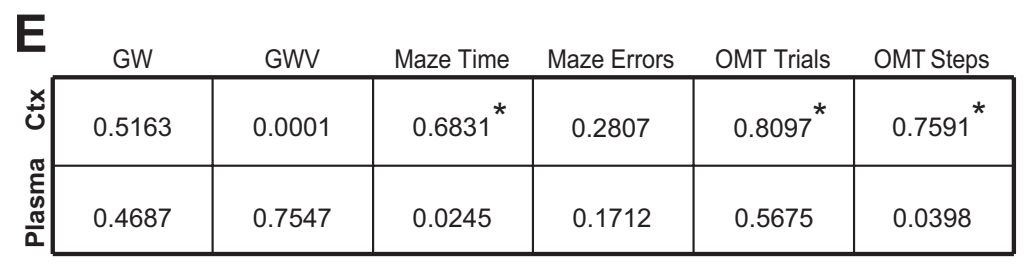

Figure 6. Viral burden is associated with neuroimmune activation and neurobehavioral performance. FIV + cats showed detectable levels of virus in cortex and plasma $(\boldsymbol{A})$. Regression analyses of $C D \boldsymbol{\varepsilon}(\boldsymbol{B}), F 4 / 80(\boldsymbol{C})$, and GFAP (D) transcript levels compared with cortical viral load. Linear regression analysis of neurobehavioral task performance (gait width, gait width variance, maze completion time, maze navigation errors, successful object memory trials, and object memory step heights) with cortex and plasma viral burden revealed significant $r^{2}$ values in cortical viral load $(\boldsymbol{E})$. Copy numbers in blood and brain were derived from a standard curve: $y=-3.34+43.1$; correlation coefficient, 0.999; PCR efficiency, 99.2\%. ${ }^{*} p<0.05$.

\section{Discussion}

Herein, we show that FIV-infected cats exhibit systemic immunosuppression that was accompanied by quantifiable neurobehavioural deficits involving both motor and cognitive dysfunc- tions. The present study represents the first quantitative analyses of neurobehavioral performance in a lentivirus model of HIV/ AIDS. Indeed, the current findings recapitulate observations in HIV-infected humans in that ataxia was evident in terms of in- 

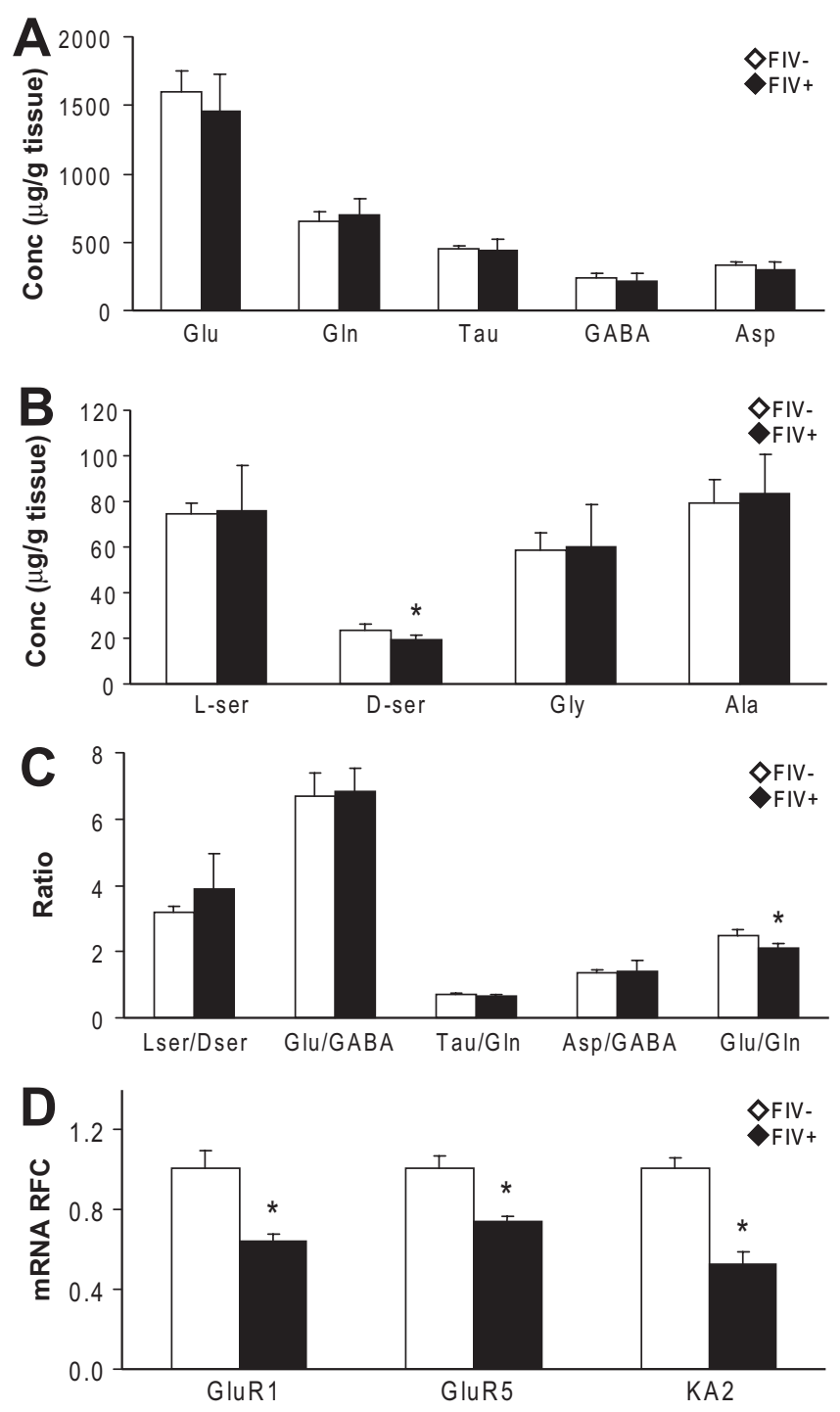

Figure 7. Cortical amino acid levels and glutamate receptor expression in FIV infection. Mean amino acid levels in brain including glutamate, glutamine, taurine, GABA, and aspartic acid $(\boldsymbol{A})$, and L-serine, D-serine, glycine, alanine $(\boldsymbol{B})$ were measured in cortex of experimental groups, although only D-serine showed a significant difference between FIV + and FIVgroups. $C$, Mean amino acid ratios disclosed that glutamate/glutamine ratios were reduced in FIV + animals. D, Transcript levels for GluR1, GluR5, and KA5 showed a reduction in FIV + animals. Shown are mean \pm SD. ${ }^{*} p<0.05$.

creased gait width variation among FIV-infected animals together with impaired learning in the maze assay (Berger and Arendt, 2000). Moreover, we show that a cortical motor recall task, the object memory test (OMT), was also impaired in the FIV-infected animals. Neurobehavioral function was inversely correlated with neuroimmune activation involving macrophage/ microglia as well as T-cell presence but not with astrocyte expression. Viral burden in cortex was also correlated with neurobehavioral performance and cellular activation. Indeed, the neurobehavioral deficits were accompanied by with neuronal loss in the parietal cortex and hippocampus and suppression of glutamate receptor expression. Hence these observations emphasize the tightly integrated relationships between neurobehavioral deficits associated with lentivirus infections and neuroinflammation, viral abundance with ensuing neurodegeneration.

Dysfunction in both gait and cognition are among the greatest disabilities experienced by patients with HIV/AIDS. The timed gait test is a useful measure of motor capacity in patients (Robertson et al., 2006) but is often affected by tissue injury at multiple sites within the nervous system during HIV infection, including peripheral nerve, spinal cord, or within the cerebellum or cerebrum. The present findings indicated that, although gait width was greater in FIV-infected animals, the variation in gait was a more robust gauge of disability and might in part reflect the outbred nature of the present host species. Similarly, the cognitive component of the maze task, indicated by the number of errors, tended to show more consistent differences between the FIV - and FIV+ groups, emphasizing neurocognitive dysfunction in this model. Thus, these observations point to neurological injury affecting multiple sites within the nervous system, in keeping with the recognition that FIV, like HIV and SIV, is able to infect all levels of the neural axis.

Like the gait and maze tasks, there are two components measured in the object memory test, including the frequency of successful trials and the percentage of steps that clear the barrier. These tasks are comparatively complex but likely indicate deficits in parietal cortex processing, based on our previous studies (McVea and Pearson, 2006). The parietal cortex is known to be involved in reprocessing the memory of object location, and neurons in areas of the posterior parietal cortex have activity patterns consistent with a role in this function (Lajoie et al., 2007). In fact, the results of the present studies suggest that the impairment observed in FIV-infected animals is not merely developmental delay but actually due to ongoing neurodegeneration because the FIV-infected group exhibit a sustained worsening in performance over time (12 and 15 weeks after infection). Of interest, the parietal cortex is adversely affected by HIV infection, as evidenced by neuroinflammation and neuronal loss, similar to the present findings (Bell et al., 2006). The object memory task is a cortical measure of neurobehavioral function and was the most robust task in distinguishing FIV - from FIV + animals. The object memory task was also correlated with neuroinflammation, and viral burden likely because of the preeminent role of the cerebral cortex in behavior. This relatively stereotyped measure of object memory function might lend itself to additional analysis in humans using functional magnetic resonance imaging and positron emission tomographic imaging in the future (Wolbers et al., 2008).

A key assumption in the lentivirus neuropathogenesis literature is that neuroinflammation and neuronal loss underlie the clinical manifestation of HIV-associated neurocognitive disorder (Reynolds et al., 2007). To test this hypothesis, we attempted to correlate neurobehavioral performance with the relative expression of several cellular markers that are induced during neuroinflammatory conditions. The macrophage/microglial marker, F4/ 80, was transcriptionally induced in FIV-infected brains and was negatively correlated with performance on multiple neurobehavioral tests. Likewise, transcript abundance of the T-cell marker, $\mathrm{CD} 3 \varepsilon$, was increased in the brains of FIV-infected animals and inversely associated with neurobehavioral function. However, the astrocyte marker, GFAP, was not activated transcriptionally by FIV infection and nor was its transcript expression associated in any way with neurological function. Indeed, viral copy number in cortex, but not plasma, was also associated with CD3 $\varepsilon$ abundance and performance of neurobehavioral tasks. Thus, these findings provide a defined link between neurobehavioral changes with brain inflammation and viral burden, which has not been previously reported as quantitative and correlative parameters.

Previous studies of lentivirus infections of the nervous system have highlighted the neuropathological consequences of infec- 
tion, consistently showing activation of perivascular macrophages and resident microglia. Analogous changes in the brain during FIV infection were apparent in the present studies, including upregulation of the macrophage/microglial marker, Iba-1, as measured qualitatively. Using the same methods, we also demonstrate that the astrocyte marker, GFAP, remained unchanged. Moreover, neuronal loss was evident in the parietal cortex and hippocampus of FIV-infected animals, which reinforces the current molecular observations. The neuronal dropout was quantified to reinforce the qualitative neuropathological observations, thereby yielding the greatest differences between FIV + and FIV - animals in the parietal cortex, which is in agreement with the outcomes in the object memory test. Thus, these results serve to underline the close relationship interlocking neurobehavioral performance, neuroinflammation, and neuronal viability in a quantitative manner.

Levels of amino acid neurotransmitters are used as a marker of neuronal injury in neurodegenerative diseases and neurological disorders. Although we found that FIV infection did not affect the levels of glutamate, GABA, and aspartate similar to a previous study using proton magnetic resonance spectroscopy (Cloak et al., 2004), the reduction of the glutamate/glutamine ratio, an index of glutamate-glutamine cycle, indicates that FIV infection affects glutamatergic neurotransmission. Moreover, the trend in reduced glutamate levels together with lower expression of AMPA and kainate receptors might further support the evidence of neuronal loss. This decrease in expression of glutamate receptors in our model is also consistent with the lower expression of AMPA and NMDA receptors in HIV-infected patients (Everall et al., 1995; Sardar et al., 1999). Although D-serine is a coactivator of NMDA receptors and has been speculated to play a role in several neurological disorders such as schizophrenia, Alzheimer's disease, and amyotrophic lateral sclerosis (Yoshimura and Goto, 2008), the change in $\mathrm{D}$-serine levels in brain has not been related to lentiviral infection and additional investigation of $\mathrm{D}$-serine might yield more insight into its contribution to lentivirus neuropathogenicity.

The present model of HIV/AIDS has the greatest application to pediatric HIV/AIDS as it is a perinatal infection model, similar to what is observed in HIV-infected children (Van Rie et al., 2007). Hence an obvious strategy will be to extend the present findings to the clinical circumstances targeting the pediatric population with HIV infection, a group mostly neglected to date in terms of understanding the associated neuropathogenic mechanisms. Nevertheless, a model that unifies neuropathology, neuroinflammation, viral burden, neurobehavioral performance, and neuronal viability in the context of a naturally occurring infection represents a unique tool for understanding disease mechanisms at any age.

\section{References}

Ances BM, Ellis RJ (2007) Dementia and neurocognitive disorders due to HIV-1 infection. Semin Neurol 27:86-92.

Bell JE, Anthony IC, Simmonds P (2006) Impact of HIV on regional and cellular organisation of the brain. Curr HIV Res 4:249-257.

Bendinelli M, Pistello M, Lombardi S, Poli A, Garzelli C, Matteucci D, Ceccherini-Nelli L, Malvaldi G, Tozzini F (1995) Feline immunodeficiency virus: an interesting model for AIDS studies and an important cat pathogen. Clin Microbiol Rev 8:87-112.

Berger JR, Arendt G (2000) HIV dementia: the role of the basal ganglia and dopaminergic systems. J Psychopharmacol 14:214-221.

Cloak CC, Chang L, Ernst T, Barr MC, Huitron-Resendiz S, Sanchez-Alavez M, Phillips TR, Henriksen S (2004) Methamphetamine and AIDS: 1HMRS studies in a feline model of human disease. J Neuroimmunol 147:16-20.

Everall IP, Hudson L, al-Sarraj S, Honavar M, Lantos P, Kerwin R (1995) Decreased expression of AMPA receptor messenger RNA and protein in AIDS: a model for HIV-associated neurotoxicity. Nat Med 1:1174-1178

Fletcher NF, Brayden DJ, Brankin B, Callanan JJ (2008) Feline immunodeficiency virus infection: a valuable model to study HIV-1 associated encephalitis. Vet Immunol Immunopathol 123:134-137.

Grant SL, Shulman Y, Tibbo P, Hampson DR, Baker GB (2006) Determination of D-serine and related neuroactive amino acids in human plasma by high-performance liquid chromatography with fluorimetric detection. J Chromatogr B Analyt Technol Biomed Life Sci 844:278-282. 
Johnston JB, Power C (2002) Feline immunodeficiency virus xenoinfection: the role of chemokine receptors and envelope diversity. J Virol 76:3626-3636.

Johnston JB, Silva C, Power C (2002) Envelope gene-mediated neurovirulence in feline immunodeficiency virus infection: induction of matrix metalloproteinases and neuronal injury. J Virol 76:2622-2633.

Kaul M, Lipton SA (2006) Mechanisms of neuroimmunity and neurodegeneration associated with HIV-1 infection and AIDS. J Neuroimmune Pharmacol 1:138-151.

Lajoie K, Andujar J, Pearson KG, Drew T (2007) Persistent neuronal activity in posterior parietal cortex area 5 related to long-lasting memories of obstacles in walking cats. Soc Neurosci Abstr 33:397.8.

Levine MS, Lloyd RL, Fisher RS, Hull CD, Buchwald NA (1987) Sensory, motor and cognitive alterations in aged cats. Neurobiol Aging 8:253-263.

McArthur JC, Brew BJ, Nath A (2005) Neurological complications of HIV infection. Lancet Neurol 4:543-555.

McVea DA, Pearson KG (2006) Long-lasting memories of obstacles guide leg movements in the walking cat. J Neurosci 26:1175-1178.

Meeker RB (2007) Feline immunodeficiency virus neuropathogenesis: from cats to calcium. J Neuroimmune Pharmacol 2:154-170.

Murray EA, Rausch DM, Lendvay J, Sharer LR, Eiden LE (1992) Cognitive and motor impairments associated with SIV infection in rhesus monkeys. Science 255:1246-1249.

Noorbakhsh F, Tang Q, Liu S, Silva C, van Marle G, Power C (2006) Lentivirus envelope protein exerts differential neuropathogenic effects depending on the site of expression and target cell. Virology 348:260-276.

Patrick MK, Johnston JB, Power C (2002) Lentiviral neuropathogenesis: comparative neuroinvasion, neurotropism, neurovirulence and host neurosusceptibility. J Virol 76:7923-7931.

Phillips TR, Prospéro-García O, Puaoi DL, Lerner DL, Fox HS, Olmsted RA, Bloom FE, Henriksen SJ, Elder JH (1994) Neurological abnormalities associated with feline immunodeficiency virus infection. J Gen Virol 75:979-987.

Poli A, Pistello M, Carli MA, Abramo F, Mancuso G, Nicoletti E, Bendinelli M (1999) Tumor necrosis factor-alpha and virus expression in the central nervous system of cats infected with feline immunodeficiency virus. J Neurovirol 5:465-473.

Power C (2001) Retroviral diseases of the nervous system: pathogenic host response or viral gene-mediated neurovirulence? Trends Neurosci 24:162-169.

Power C, Moench T, Peeling J, Kong PA, Langelier T (1997) Feline immunodeficiency virus causes increased glutamate levels and neuronal loss in brain. Neuroscience 77:1175-1185.

Power C, Buist R, Johnston JB, Del Bigio MR, Ni W, Dawood MR, Peeling J (1998) Neurovirulence in feline immunodeficiency virus-infected neonatal cats is viral strain specific and dependent on systemic immune suppression. J Virol 72:9109-9115.

Power C, Gill MJ, Johnson RT (2002) The neuropathogenesis of HIV infec- tion: host-virus interaction and the impact of therapy. Can J Neurol Sci 29:19-32.

Power C, Henry S, Del Bigio MR, Larsen PH, Corbett D, Imai Y, Yong VW Peeling J (2003) Intracerebral hemorrhage induces macrophage activation and matrix metalloproteinases. Ann Neurol 53:731-742.

Prospéro-García O, Huitrón-Resendiz S, Casalman SC, Sánchez-Alavez M, Díaz-Ruiz O, Navarro L, Lerner DL, Phillips TR, Elder JH, Henriksen S (1999) Feline immunodeficiency virus envelope protein (FIVgp120) causes electrophysiological alterations in rats. Brain Res 836:203-209.

Reynolds A, Laurie C, Mosley RL, Gendelman HE (2007) Oxidative stress and the pathogenesis of neurodegenerative disorders. Int Rev Neurobiol 82:297-325.

Robertson KR, Parsons TD, Sidtis JJ, Hanlon Inman T, Robertson WT, Hall CD, Price RW (2006) Timed gait test: normative data for the assessment of the AIDS dementia complex. J Clin Exp Neuropsychol 28:1053-1064.

Ryan G, Grimes T, Brankin B, Mabruk MJ, Hosie MJ, Jarrett O, Callanan JJ (2005) Neuropathology associated with feline immunodeficiency virus infection highlights prominent lymphocyte trafficking through both the blood-brain and blood-choroid plexus barriers. J Neurovirol 11:337-345.

Sardar AM, Hutson PH, Reynolds GP (1999) Deficits of NMDA receptors and glutamate uptake sites in the frontal cortex in AIDS. Neuroreport 10:3513-3515.

Steigerwald ES, Sarter M, March P, Podell M (1999) Effects of feline immunodeficiency virus on cognition and behavioral function in cats. J Acquir Immune Defic Syndr Hum Retrovirol 20:411-419.

Tsutsui S, Schnermann J, Noorbakhsh F, Henry S, Yong VW, Winston BW Warren K, Power C (2004) Al adenosine receptor upregulation and activation attenuates neuroinflammation and demyelination in a model of multiple sclerosis. J Neurosci 24:1521-1529.

Van Rie A, Harrington PR, Dow A, Robertson K (2007) Neurologic and neurodevelopmental manifestations of pediatric HIV/AIDS: a global perspective. Eur J Paediatr Neurol 11:1-9.

Wolbers T, Hegarty M, Büchel C, Loomis JM (2008) Spatial updating: how the brain keeps track of changing object locations during observer motion. Nat Neurosci 11:1223-1230.

Yoshimura T, Goto M (2008) D-Amino acids in the brain: structure and function of pyridoxal phosphate-dependent amino acid racemases. FEBS J 275:3527-3537.

Zhu Y, Jones G, Tsutsui S, Opii W, Liu S, Silva C, Butterfield DA, Power C (2005) Lentivirus infection causes neuroinflammation and neuronal injury in dorsal root ganglia: pathogenic effects of STAT-1 and inducible nitric oxide synthase. J Immunol 175:1118-1126.

Zhu Y, Antony JM, Martinez JA, Glerum DM, Brussee V, Hoke A, Zochodne D, Power C (2007) Didanosine causes sensory neuropathy in an HIV/ AIDS animal model: impaired mitochondrial and neurotrophic factor gene expression. Brain 130:2011-2023

Zink MC, Laast VA, Helke KL, Brice AK, Barber SA, Clements JE, Mankowski JL (2006) From mice to macaques - animal models of HIV nervous system disease. Curr HIV Res 4:293-305. 